\title{
The Case for Antiparticles in the Extragalactic Cosmic Radiation
}

F.W. Stecker and A.W. Wolfendale

JANUARY 1984

Natıonal Aeronautics and

Space Adminıstratıon

Goddard Space Flight Center

Greenbelt, Maryland 20771 
THE CASE FOR ANTIPARTICLES IN THE EXTRAGALACTIC COSMIC RADIATION

\author{
F.W. Stecker \\ Laboratory for High Energy Astrophysics \\ NASA Goddard Space Flight Center \\ Greenbelt, Maryland, U.S.A.
}

and

A.W. Wolfendale

Physics Department

University of Durham

Durham, U.K. 
The presence of an excess of low energy antiprotons in the primary cosmic radiation $^{1-3}$ has given rise to a number of possible explanations 4 , some of which involve exotic processes such as mini black holes and extragalactic antiparticles. We consider here the last mentioned possibility 5 and show that there are interesting implications for the bulk of the cosmic radiation at higher energies. Indeed, it may be possible to account for a previously puzzling feature - a "bump" in the energy range $10^{14}-10^{15} \mathrm{eV}$ - of the cosmic ray spectrum by hypothesizing a primary extragalactic origin for the bulk of the observed cosmic ray antiprotons, although such an explanation is not unique. In this model, most of the cosmic rays above $10^{15} \mathrm{eV}$ are extragalactic. We describe a method of testing this hypothesis experimentally.

One of the most fundamental questions in cosmology is the question of the existence of antimatter in significant quantities in the universe. Does antimatter play an equal role with matter in the makeup of the galaxies? This question has now become a question of fundamental importance to physics as well. In the contemporary paradigm of grand unified gauge theories it is related to the question of the nature of CP violation at high energies ${ }^{6}$. Early attempts at constructing a baryon symmetric cosmology 7,8 suffered from the difficulty of providing an effective mechanism for separating matter and antimatter on ultimately large enough scales to be consistent with astronomical observations. However, recent theoretical work based on the concepts of grand unified theories have resulted in the development of a plausible baryon-antibaryon domain theory in which matter and antimatter are created in separate regions of survivable size to begin with ${ }^{9-12}$. Various observational aspects of this theory have been previously discussed 13,14 and the subject of baryon symmetric cosmology has been recently reviewed 
elsewhere 15,16 . It should, of course, be noted that regardless of the ultimate fate of our present theoretical ideas either in favor of or against the reality of baryon symmetry on an overall universal scale, the experimental search for primary cosmologically significant quantities of antimatter is a task of deep significance. And it can further be said that both the recent theoretical work and the reports by various groups of surprisingly large fluxes of antiprotons in the cosmic radiation give immediacy to this search.

The present status of cosmic ray antiproton measurements and the attempts to understand them have been recently reviewed 4 and an exegesis of the primary extragalactic origin hypothesis has also been recently given ${ }^{5}$. We will discuss further implications of potential basic import to cosmic ray research here and we will also propose an experimental search program based on these considerations. We start with the hypothesis that the baryon symmetric domain cosmology leads to a flux of extragalactic cosmic rays consisting of roughly equal amounts of protons and antiprotons with the sources of these cosmic rays being primarily active galaxies 17,18 and with helium and antihelium nuclei being supressed by destruction processes in these sources 5 . (We will also assume that helium and antihelium nuclei exist in the extragalactic cosmic rays at a lower level, supplied by leakage from normal matter galaxies and normal antimatter galaxies.) We further assume that the galactic wind is too weak to keep out the extragalactic cosmic radiation. Observations favor the interpretation that the galactic wind is in reality a "breeze"19.

Let us first consider the energy spectrum of the cosmic radiation. The measured spectrum of cosmic radiation can be represented by a power 1 aw in energy of the form $\mathrm{KE}^{-\Gamma}$ with the spectral index $\Gamma \simeq 2.75$ for several decades above the $10 \mathrm{GeV}$ energy level. It appears likely that this radiation is produced primarily in galactic sources 20,21. Furthermore, the source 
spectrum of this radiation is expected to have a lower spectral index $\Gamma_{S}$ than that observed at the earth which has been steepened by energy dependent propagation effects. A value for $\Gamma_{S}$ of approximately 2.0 to 2.2 appears to be likely for two reasons. (1) Measurements of the ratio of secondary to primary nuclei in the cosmic radiation suggest that the mean lifetime in the Galaxy owing to trapping by the tangled galactic magnetic fields falls with energy as $E^{-\delta}$ where the most recently derived value 22 of $\delta \simeq 0.7$. (2) The theoretical shock acceleration models for cosmic ray production currently favored 23 generally yield production spectra with $\Gamma_{S}$ close to 2 .

If we assume that there exists a general acceleration mechanism for generating cosmic rays which acts in both galactic and extragalactic sources to give a universal source spectrum with $\Gamma_{S} \simeq 2$, as is now thought to be the case with shock acceleration, then the extragalactic cosmic ray component should reflect this source spectrum. Thus, with the antiprotons assumed to be both primary and extragalactic and the bulk of the protons to be galactic, the expected ratio of antiprotons to protons should increase with energy as $\mathrm{E}^{\delta}$. Taking $\delta \simeq 0.7$, antiprotons could make up approximately one per cent of the cosmic ray flux at an energy of $\simeq 500 \mathrm{GeV}$ and even $\sim 50$ per cent at higher energies. This has important observational implications which we will discuss later. The situation is indicated in Figure 1 which shows the present data on antiproton intensities and a theoretical extrapolation corresponding to a primary extragalactic antiproton flux with $\Gamma=2.0$. For these considerations, we assume that secondary production of antiprotons is unimportant. (See, e.g., the estimate given in Figure 1).

Figure 2 shows the effect of extrapolating the extragalactic intensity of both protons and antiprotons (this introduces a factor of two) with a spectral index of 2 to higher energies and superposing it on the galactic cosmic ray 
spectrum with index $\Gamma \simeq 2.75$. Note that such an extrapolation implies that the extragalactic and galactic cosmic ray fluxes become comparable at an energy of about $10^{5} \mathrm{GeV}$ and that extragalactic particles predominate above this energy. It is interesting to note that the resultant flattening in the spectrum occurs at this particular energy where there have been claims 24 of a flattening in the cosmic ray spectrum as inferred from measurements of extensive air showers. It should, of course, be noted that a steepening in the spectra of both the galactic and extragalactic components would be required by the observations for energies above $10^{6} \mathrm{GeV}$, but this is not difficult to explain.

Since our model indicates that the antiproton-to-proton ratio should which increase with energy, measurements of the sign of the charges of cosmic rays at the highest practical energy and the determination of the spectra of the various charged components of the cosmic radiation up to that energy will provide a test of our hypothesis. Such a test requires the placement of the experiment above the atmosphere so that the incoming cosmic ray nuclei can be measured directly. Furthermore, the sign of their charges (and magnitude) may be measured by use of a superconducting magnet. Such an experiment, with an attainable energy of about 500 to $1000 \mathrm{GeV}$, could be flown aboard a space shuttle 25 . In addition, an emulsion stack experiment could be flown on a high altitude balloon or on the space shuttle to look for antihelium nuclei, even at the reduced level implied by our hypothesis. A polar or near polar orbit would be desirable to avoid the geomagnetic cutoff. In view of the almost impossible odds of creating a secondary ${ }^{4} \mathrm{He}$ antinucleus, the unambiguous detection of even one such particle would provide irrefutable evidence of primary cosmic ray antimatter. (It should also be noted that the production of 100-300 MeV antiprotons is extremely difficult because of well-known 
kinematical factors ${ }^{5}$. This feat has proved elusive even to accelerator physicists who have deliberately set out in the laboratory to make such particles (C. Rubbia, private communication). Thus, the observed low-energy antiprotons in the cosmic radiation are also quite difficult to explain as secondaries from cosmic-ray interactions.)

The experimental program proposed above will add importantly to our basic understanding of $\bar{p}$ origin. If the $\bar{p} / p$ ratio is observed to increase as $E^{0.7}$ or thereabouts, then our hypothesis of extragalactic antiprotons from antimatter galaxies will have very strong support. The observation of antihelium nuclei would, as already mentioned, provide certainty. The extent to which non-observation of antihelium disproves our hypothesis is unclear, but if $\bar{\alpha} / \alpha \ll 10^{-5}$ (the value expected very approximately on the basis of $\bar{\alpha}^{\prime}$ s leaking from "normal" antimatter galaxies) then the difficulty would be severe.

Non-observation of antihelium (at the level given above), together with an increase in the $\bar{p} / p$ ratio with energy at a slower rate than $E^{\delta}$ (e.g. $\alpha E^{0.25}$ between 10 and $100 \mathrm{GeV}$ ), would be consistent with the closed galaxy model (or source trapping), although there are severe theoretical problems 5,26 with such a model.

Finally, the observation of a $\bar{p} / p$ ratio falling rapidly with increasing energy would give support to the black hole model of Kiraly et al.26 (see Fig. 1).

\section{Acknowledgement}

The authors would like to thank Dr. Jonathan Ormes for helpful discussions. 


\section{REFERENCES}

1. Golden, R.L., et al., Phys. Rev. Lett. 43, 1196 (1979).

2. Bogolmolov, E.A. et al., Proc. 16th Intl. Cosmic Ray Conf. 1, 330 (1979).

3. Buffington, A. et al., Astrophys. J. 248, 1179 (1981).

4. See review by R.J. Protheroe in Composition and Origin of Cosmic Rays ed. M.M.Shapiro, D. Reidel Pub. Co., Dordrecht, Holland 119 (1983)

5. Stecker, F. W., R.J. Protheroe and D.Kazanas, Astrophys and Space Sci. $\underline{96}, 171(1983)$

6. Brown, R. W. and Stecker, F. W., Phys. Rev. Lett. 43, 315 (1979).

7. Alfven, H., Rev. Mod. Phys. 37,652 (1965).

8. Omnes, R., Phys. Rpts. 3, 1 (1972).

9. Senjanovic, G. and Stecker, F. W., Phys. Lett. 96B, 285 (1980).

10. Sato, K., Phys. Lett. 998, 66 (1981).

11. Kuzmin, V.A., Tkachev, I.I. and Shaposhnikov, M.E., Phys. Lett. 105B, 167 (1981).

12. Mohanty, A. K. and Stecker, F. W., to be published.

13. Stecker, F.W., Ann. N.Y. Acad. Sci. (Proc. 10th Texas Symp. on Relativistic Astrophys.) 375, 69 (1981).

14. Stecker, F.W., in Progress in Cosmology, ed. A.W. Wolfendale, Reidel Pub. Co., Dordrecht, Holland, 1 (1982).

15. Chechetkin, V.M., Khlopov, M. Yu. and Sapozhnikov, M. G., Rev. del Nuovo Cimento 5, 1 (1982).

16. Stecker, F.W., in Early Evolution of the Universe and its Present Structure, ed. G.0. Abell and G. Chincarini, Reidel Pub. Co., Dordrecht, Holland, 437 (1983). 
17. Ginzburg, V. L. and Syrovatskii, The Origin of Cosmic Rays, Pergamon Press, London (1964).

18. Hayakawa, S. Cosmic Ray Physics, Wiley \& Sons, New York (1969).

19. Jones, F.C., Astrophys. J. 229,747 (1979).

20. Codds, D. Strong, A.W. and Wolfendale, A.W., Mon. Not. Royal Astron. Soc. $171,569(1975)$.

21. Stecker, F.W., Phys. Rev. Lett. 35, 188 (1975).

22. Ormes, J.F. and Protheroe, R.J., Astrophys. J. 272, 756 (1983).

23. Drury, I., Axford, W.I. and Summers, D., Mon. Not. Royal Astron. Soc. 198, $833(1982)$.

24. Kempa, J, Wdowczyk, J. and Wolfendale, A.W., J. Phys. A 7, 1213 (1974).

25. SUPERMAG proposal, T. Bowen, et al. (1979).

26. Kiraly, P., et al., Nature 293, 120 (1981). 
- Figure Captions

Figure 1. Observations and theoretical $\bar{p}$ spectra. The data points are: Bfrom Buffington et al. (Ref. 3), Bo-from Bogomolov, et al. (Ref. 2) and G- from Golden, et al. (Ref. 1). The higher points marked B take account of solar modulation effects (Ref, 26). The solid curve $(S)$ is for secondary $\bar{p}$ production model with mean propagation length $5 \mathrm{~g} \mathrm{~cm}^{-2}$ and the dashed line corresponds to the shape of the $\bar{p}$ spectrum calculated for a primordial black hole model (Ref. 26). The higher solid line corresponds to our spectrum for the extragalactic primary hypothesis as discussed in the text.

Figure 2. The effect of extragalactic primary protons and antiprotons on the total cosmic ray spectrum according to the model discussed in the text. It can be seen that this model may account for the putative flattening in the observed cosmic ray spectrum near $10^{14} \mathrm{eV}$. 


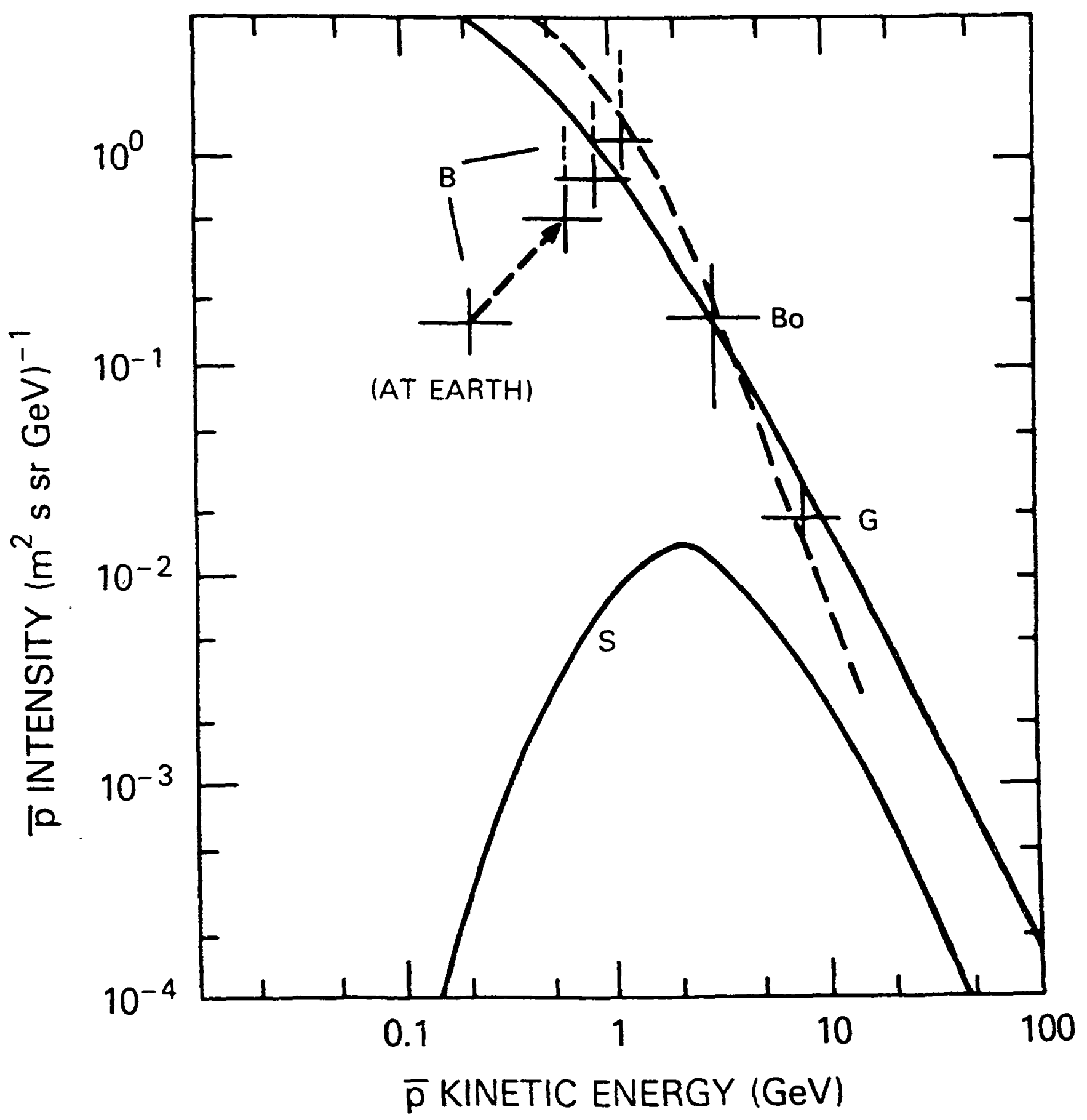

Fig. 1 


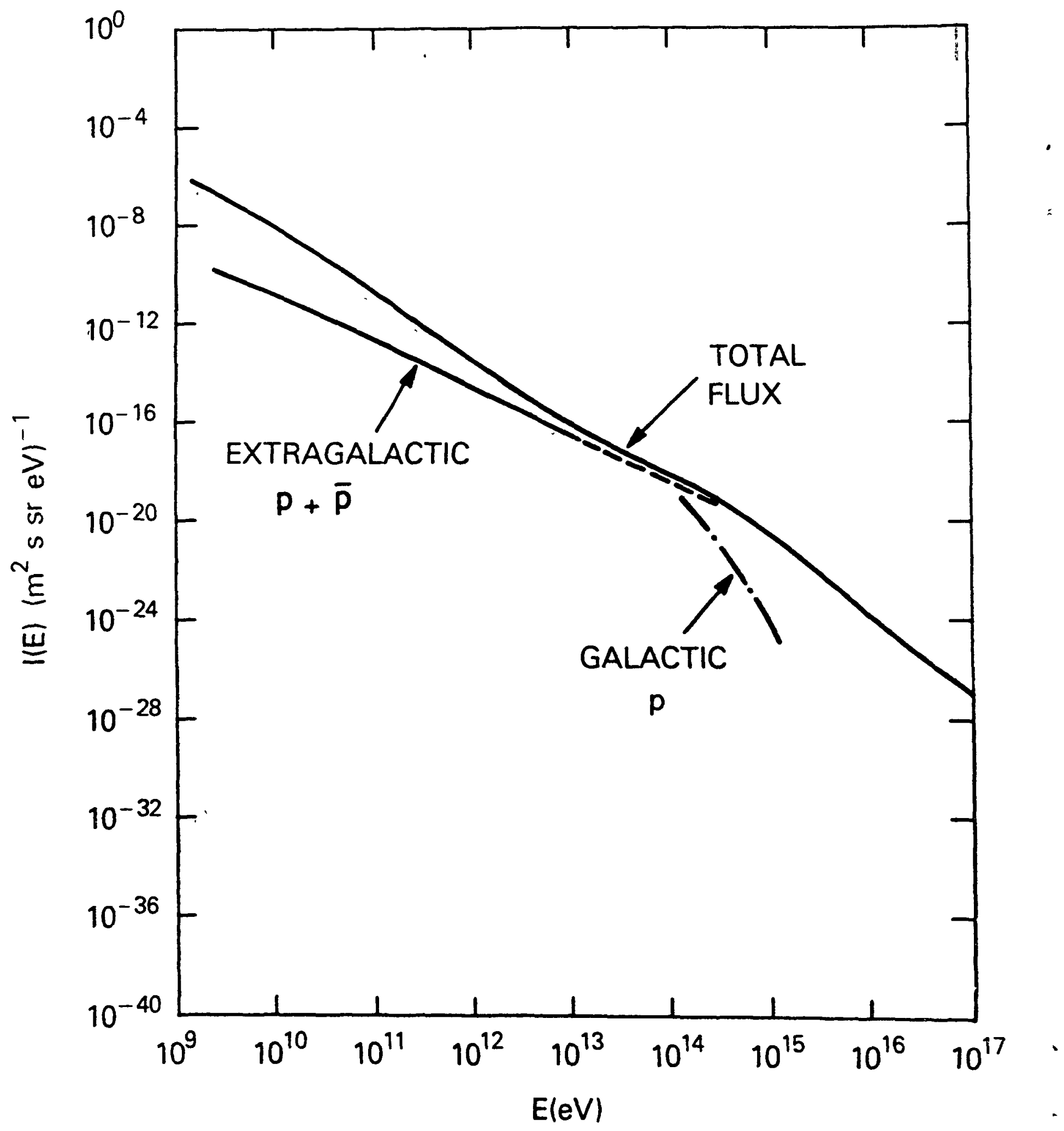

Fig. 2 
BIBLIOGRAPHIC DATA SHEET

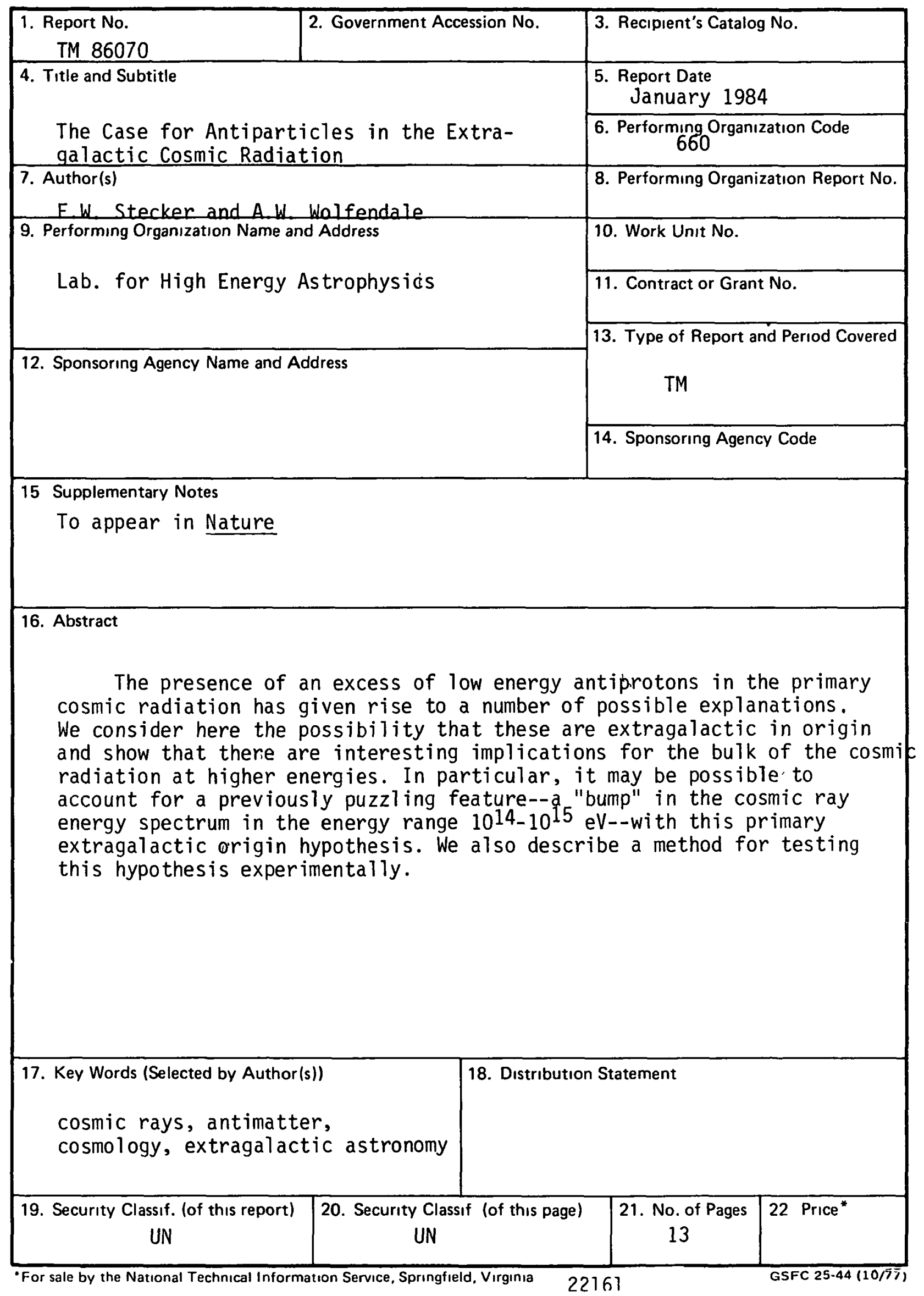

